\title{
Cambios histológicos dérmicos tras pérdida masiva de peso y su repercusión en la herida quirúrgica en abdominoplastia
} Histological dermal changes after massive weight loss and its impact on surgical wound after abdominoplasty

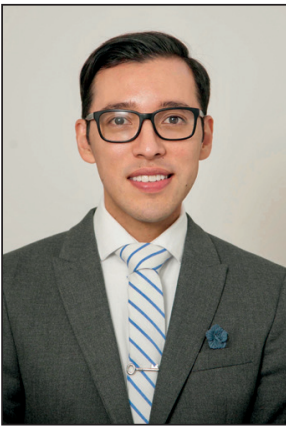

Morales Olivera J.M.

\section{Resumen}

Introducción y Objetivo. La obesidad es un problema de salud pública mundial. Tras la cirugía metabólica se genera una pérdida masiva de peso tras la cual suele ser necesaria una cirugía reconstructiva que conlleva un alto porcentaje de complicaciones en el sitio quirúrgico, principalmente dehiscencia.

Es necesario evaluar los cambios histológicos que sufren estos pacientes y su relación con la evolución de la herida, marcando las diferencias entre los pacientes que pierden peso con dieta y ejercicio (únicamente con sobrepeso previo), en comparación con aquellos sometidos a cirugía bariátrica.

Material y método. Estudiamos 4 grupos de pacientes candidatos a abdominoplastía tras pérdida de peso hasta lograr un índice de masa corporal (IMC) óptimo. Grupo A (grupo control): pacientes con sobrepeso (IMC 25.1-29) y pérdida ponderal solo con dieta y ejercicio hasta IMC aceptado $<27.5$. Grupo B: obesidad Grado I (IMC 30-34.9); Grupo C: obesidad Grado II (IMC 35-39.9); Grupo D: obesidad Grado III / obesidad mórbida (IMC $>40$ ). Los Grupos B, C y D con pérdida ponderal tras bypass gástrico que llevó a un IMC aceptado $<31$.

Tomamos biopsia de la porción supraumbilical del colgajo abdominal a resecar y analizamos las características histológicas y su relación con la evolución postoperatoria.

Resultados. Incluimos 80 pacientes ( 20 por grupo), 95\% mujeres ( 76 pacientes), con edad promedio de 39.4 años (rango de 29 a 58). Obtuvimos una $\mathrm{p}<0.0001$ en relación a la densidad y morfología de las fibras elásticas y de colágeno al comparar todos los grupos de obesidad con el grupo control (sobrepeso); un riesgo relativo 5 veces mayor de equimosis y epidermólisis en la obesidad GIII; 8 veces más riesgo de dehiscencia en la obesidad GII; y 14 veces más riesgo de dehiscencia en el GIII. No hubo diferencia estadísticamente significativa para infección.

Conclusiones. Los pacientes con mayor grado de obesidad (GII y III) presentan mayores alteraciones histológicas dérmicas, con repercusión directamente proporcional (aumento del riesgo relativo) en la presencia de equimosis, epidermólisis y dehiscencia de las heridas. Recomendamos tener conciencia de este fenómeno al realizar procedimientos quirúrgicos en pacientes con pérdida de peso tras bypass gástrico, y en el momento del cierre quirúrgico, aplicar menor tensión en los bordes de la herida.

Palabras clave $\begin{aligned} & \text { Obesidad, Pérdida masiva peso, } \\ & \text { Cirugía bariátrica, Cirugía postbariátrica }\end{aligned}$
$\begin{array}{lr}\text { Nivel de evidencia científica } & 3 c \text { Terapéutico } \\ \text { Recibido [esta versión] } & 20 \text { abril/2019 } \\ \text { Aceptado } & 30 \text { julio/2019 }\end{array}$

Background and Objective. Obesity is a global public health problem. Metabolic surgery causes a massive weight loss, and patients usually require reconstructive surgery with a high percentage of complications at the surgical site, mainly dehiscence.

Consequently, an analysis of histological changes on these patients and their relationship with wound evolution is required, stressing the differences between a patient who lost weight with diet and exercise (with prior overweight only), compared to one subjected to bariatric surgery.

Methods. We formed 4 groups of patients who would undergo abdominoplasty after weight loss until an optimal body mass index (BMI) was obtained. Group A (control group): patients who were overweight (BMI 25.1-29), losing weight only with diet and exercise, leading them to an acceptable BMI $<27.5$. Group B: obesity Grade I (BMI 30-34.9). Group C: obesity Grade II (BMI 35-39.9). Group D: obesity Grade III / morbid obesity (BMI $>40$ ). Groups B, C and D with weight loss after gastric bypass, leading to an acceptable BMI $<30$. A biopsy was taken from the supraumbilical portion of the abdominal flap to be resected. Histological characteristics and their relationship with postoperative evolution were analyzed.

Results. 80 patients were included ( 20 per group). 95\% were female (76 patients), with an average age of 39.4 years old (range from 29 to 58). A $p<0.0001$ was obtained in relation to density and morphology of elastic and collagen fibers when comparing all obesity groups to the control group (overweight), as well as a relative risk 5 times higher of having ecchymosis and epidermolysis in obesity GIII; 8 times higher risk of having dehiscence in Obesity GII; and 14 times higher risk of dehiscence in grade III. There was no statistically significant difference for infections.

Conclusions. Patients with a higher degree of obesity (GII and III) have greater dermal histological alterations, with a directly proportional impact (increased relative risk) on the presence of ecchymosis, epidermolysis and dehiscence of wounds. Therefore, we should be aware of this problem when performing surgical closure, applying less pressure on edges. We recommend to take on count this phenomenon when performing a surgical procedure on a patient who lost weight after a gastric bypass, and so, a lower pressure must be applied to closure.

\begin{tabular}{|lr|}
\hline Key words & $\begin{array}{l}\text { Obesity, Massive lost weight, } \\
\text { Bariatric surgery, Postbariatric surgery }\end{array}$ \\
Level of evidence & $3 \mathrm{c}$ Therapeutic \\
Received [this version] & 20 April/2019 \\
Accepted & 30 July/2019 \\
\hline
\end{tabular}

Conflicto de intereses: Los autores declaran no tener ningún interés financiero relacionado con el contenido de este artículo. Financiación: No hubo fuentes externas de financiación para este trabajo. 
Introducción

La obesidad es un problema de salud pública a nivel mundial. La cirugía bariátrica se ha descrito como solución definitiva a la obesidad mórbida, aquella definida como Grado III con un índice de masa corporal (IMC) mayor de $40 \mathrm{~kg} / \mathrm{m}^{2}$. Sin embargo, cuando las medidas higiénico-dietéticas no son suficientes para la disminución del peso corporal o existen comorbilidades de difícil control, la cirugía bariátrica también se puede realizar en los paciente con obesidad Grado II y III, aquellas con IMC de 30 a 34.9 y de 35 a 39.9 respectivamente. ${ }^{(1,2)}$

Tras la pérdida ponderal de peso derivada de la cirugía bariátrica se logra la resolución de la mayoría de las comorbilidades asociadas a la obesidad, ya sea mórbida o de menor grado pero de difícil control. Sin embargo, también sobrevienen los respectivos cambios estéticos a todos niveles corporales, derivados del exceso de piel redundante. ${ }^{(3)}$ Estos cambios clínicos cutáneos consisten principalmente en una gran laxitud debida a la remodelación y resorción de tejido adiposo y a la modificación de sus propiedades elásticas, provocando cambios generales en la función de barrera, en la microcirculación, en el flujo linfático, la pigmentación y causando alteración en los anexos (glándulas sebáceas y folículos pilosos). Todo ello se traduce en edema, ulceración y tendencia a patologías localizadas. ${ }^{(4.5)}$ Asimismo, existen cambios directos sobre la matriz extracelular que consisten en degradación de las fibras de elastina y depleción o cambios estructurales en las fibras de colágeno. ${ }^{(6)}$

En la piel normal, la dermis papilar contiene fibras elásticas delgadas dispuestas de forma perpendicular a la unión dermoepidérmica y conectadas a la lámina basal. Bioquimicamente, la dermis papilar está compuesta en su mayoría por colágeno tipo III y la dermis reticular por colágeno tipo I, sin embargo, su cuantificación es sumamente difícil por lo que su observación se basa más en su densidad y calidad que en valores cuantitativos. ${ }^{(8)}$

Existe la teoría de que tras una pérdida ponderal la degeneración en la dermis produce cambios en:

1) La densidad de fibras elásticas (elastina).

2) La morfología de las fibras elásticas.

3) La densidad de fibras de colágena.

4) La calidad de fibras de colágena.

5) El grosor de la epidermis (estrato córneo).

6) La actividad de los fibroblastos /inflamación.

7) La calidad de los adipocitos

(en muestras/biopsias analizadas en bloque).

Dichos cambios están en relación con el grado de tensión producida por la propia obesidad y en ello radi- ca la importancia del presente estudio: evidenciar que, a mayor obesidad, mayor deterioro de las condiciones de la piel y mayor repercusión en la evolución del sitio quirúrgico. ${ }^{(9)}$

\section{Material y método}

Llevamos a cabo un estudio descriptivo y comparativo en el que el universo de estudio estuvo compuesto por pacientes programados para abdominoplastia, ya fuera:

- Abdominoplastia convencional, cuando presentaron sobrepeso (grupo control) y lograron pérdida ponderal únicamente con medidas higiénico-dietéticas. Nos referimos a la abdominoplastia descrita como resección del colgajo abdominal anterior con plicatura de músculos rectos, reubicación del ombligo y tensión en los bordes de acuerdo al marcaje prequirúrgico con cierre en 3 planos. Siempre dejamos drenaje cerrado. ${ }^{(10,11)}$

- Abdominoplastia circular en flor de lis, para el grupo de obesidad Grados I, II y III que lograron la reducción del IMC mediante un procedimiento bariátrico (bypass gástrico). La técnica quirúrgica en este grupo es la convencional sin despegamiento de los colgajos marcados prequirúrgicamente en el área central de la incisión vertical en su unión con la horizontal (la flor de lis), con plicatura de músculos rectos, reubicación del ombligo y cierre en 3 planos libre de tensión en su totalidad. Siempre dejamos drenaje cerrado. ${ }^{(12)}$

Los criterios de inclusión en el estudio fueron:

- Pacientes de sexo femenino o masculino menores de 65 años de edad.

- Para pacientes con sobrepeso:

○ Haber presentado un IMC de entre 25 y $29.9 \mathrm{~kg} / \mathrm{m}^{2}$ (criterio de sobrepeso) en el momento de la solicitud de abdominoplastia.

○ Haber alcanzado un IMC menor de $27.5 \mathrm{~kg} / \mathrm{m}^{2}$ (en los casos que lo necesitaran), para poder ser sometidos una abdominoplastia convencional.

- Haber logrado dicha reducción de peso únicamente mediante medidas higiénico-dietéticas.

- Para pacientes con obesidad:

○ Haber presentado un IMC mayor de $30 \mathrm{~kg} / \mathrm{m}^{2}$ (criterio de obesidad) y haber sido sometidos a un bypass gástrico (por obesidad mórbida, GIII) o por comorbilidades y/o falta de respuesta al tratamiento higiénico-dietético (en GI y II).

- Para los pacientes con obesidad GI (IMC entre 30 y $34.9 \mathrm{~kg} / \mathrm{m} 2$ ), haber alcanzado un IMC de entre 25 y $28 \mathrm{~kg} / \mathrm{m}^{2}$ para poder ser sometidos a una abdominoplastia circular en flor de lis. 
- Para los pacientes con obesidad GII (IMC de entre 35 y $39.9 \mathrm{~kg} / \mathrm{m}^{2}$ ), haber alcanzado un IMC de entre 25 y $28 \mathrm{~kg} / \mathrm{m}^{2}$ para poder ser sometidos a una abdominoplastía circular en flor de lis.

- Para los pacientes con obesidad GIII / obesidad mórbida (IMC mayor de $40 \mathrm{~kg} / \mathrm{m}^{2}$ ), haber alcanzado un IMC por lo menos inferior $31 \mathrm{~kg} / \mathrm{m}^{2}$ para poder ser sometidos a una abdominoplastia circular en flor de lis.

- Comorbilidades crónico-degenerativas como diabetes mellitus, hipertensión arterial sistémica o enfermedad tiroidea, entre otras, controladas por el Servicio de Medicina Interna.

- Tener valoración por el Servicio de Nutrición Clínica documentando un peso estable por un periodo de al menos 3 meses y por Psicología, corroborando la adecuada estabilidad emocional antes del procedimiento quirúrgico.

- Pacientes que aceptaron participar en el estudio mediante la firma del consentimiento informado.

Para determinar el tamaño de la muestra empleamos cálculo de muestras para medias con un intervalo de confianza del 95\% tomando en cuenta los estudios de Fearmonti y col. ${ }^{(9)}$ y establecimos con ellos 4 grupos (Tabla I):

Tabla I. Composición de los grupos de estudio

\begin{tabular}{|c|c|c|c|}
\hline \multicolumn{4}{|c|}{ 4 GRUPOS (n=20 cada grupo / total= 80 pacientes) } \\
\hline GRUPO A & GRUPO B & GRUPO C & GRUPO D \\
\hline SOBREPESO & $\begin{array}{c}\text { OBESIDAD } \\
\text { GI* }\end{array}$ & $\begin{array}{c}\text { OBESIDAD } \\
\text { GII* }\end{array}$ & $\begin{array}{c}\text { OBESIDAD } \\
\text { GIII* }\end{array}$ \\
\hline \multicolumn{4}{|c|}{ PÉRDIDA PONDERAL } \\
\hline MEDIDAS & BYPASS & BYPASS & BYPASS \\
HIGIÉNICO & GÁSTRICO & GÁSTRICO & GÁTRICO \\
\hline
\end{tabular}

*GI= Grado I; GII=Grado II; GIII= Grado III

- Grupo A: pacientes con sobrepeso y pérdida ponderal mediante medidas higiénico-dietéticas.

- Grupo B: pacientes con obesidad GI y pérdida ponderal mediante bypass gástrico.

- Grupo C: pacientes con obesidad GII y pérdida ponderal mediante bypass gástrico.

- Grupo D: pacientes con obesidad GIII y pérdida ponderal mediante bypass gástrico.

Durante la intervención, tomamos biopsia de piel de $4 \times 4 \mathrm{~cm}$ de la región supraumbilical del colgajo abdominal a resecar por ser la zona que reúne las mejores características en relación a la piel del borde inferior del colgajo cutáneo y se mantiene en la mayoría de los casos libre de dermatosis. Analizamos las características histo- lógicas mediante las tinciones de hematoxilina-eosina y tinción tricrómica de Masson.

Las variables clínicas del sitio quirúrgico a evaluar fueron:

1.- Cambios en la densidad de las fibras elásticas.

Nulos: sin cambios en la distribución normal previamente descrita.

Moderados: hay disminución de su distribución y se observa presencia en la dermis papilar.

Severos: hay disminución de su distribución y se observa presencia en la dermis reticular.

2.- Cambios en la morfología de las fibras elásticas.

Nulos: sin cambios en la distribución normal previamente descrita.

Moderados: adelgazamiento de sus haces en la dermis papilar.

Severos: adelgazamiento de sus haces en la dermis reticular.

3.- Cambios en la densidad del colágeno.

Nulos: sin cambios en la distribución normal previamente descrita.

Moderados: hay disminución de su distribución y se observa presencia en la dermis papilar.

Severos: hay disminución de su distribución y se observa presencia en la dermis reticular.

4.- Cambios en la morfología del colágeno.

Nulos: sin cambios en la distribución normal previamente descrita.

Moderados: adelgazamiento de sus haces en la dermis papilar y reticular superficial.

Severos: adelgazamiento de los haces en todo el espesor de la dermis.

5.- Grosor de epidermis (estrato córneo).

Normal: 20-30 capas celulares.

Aumentado (hipertrófico): >30 capas celulares.

Disminuido (atrófico): $<20$ capas celulares.

Las variables clínicas del sitio quirúrgico a evaluar fueron:

1.- Equimosis: depósitos de sangre extravasada debajo de la piel intacta.

2.- Epidermolisis: aparición de flictenas/ampollas en la piel causadas por isquemia o congestión vascular.

3.- Necrosis cutánea: muerte celular de la piel secundaria a isquemia, congestión o traumatismo directo.

4.-Dehiscencia de herida quirúrgica: separación o pérdida de continuidad de los bordes de una herida en el postoperatorio. En este estudio categorizamos las dehiscencias en 1 a $3 \mathrm{~cm}$, de 3 a $5 \mathrm{~cm}$ y de 5 a $10 \mathrm{~cm}$.

5.- Infección del sitio quirúrgico / operatorio: la que se desarrolla dentro de los primeros 30 días posteriores al procedimiento quirúrgico, y hasta 1 año después cuando se incluye material protésico. 
Resultados

\section{Estadística descriptiva}

Datos demográficos. Incluimos en el estudio 80 pacientes, 20 por cada grupo, el $95 \%$ de sexo femenino ( 76 pacientes), con una edad promedio de 39.4 años (rango de 29 a 58 años).

En el grupo A (pacientes con sobrepeso previo a la pérdida ponderal por medidas higiénico-dietéticas), identificamos una edad promedio de 39.2 años ( 29 a 48 años); el IMC promedio inicial fue de $29.23 \mathrm{~kg} / \mathrm{m}^{2}(27.1$ a 29.8), con una pérdida promedio de $6 \mathrm{~kg}(4.5 \mathrm{a} 8.5 \mathrm{~kg})$, alcanzando un IMC de $26.89 \mathrm{~kg} / \mathrm{m}^{2}$ (24.8 a 27.4). Solamente 1 paciente de este grupo presentó como comorbilidad hipertensión arterial sistémica controlada (Tabla II).

En el grupo B (pacientes con obesidad GI previa a la pérdida ponderal por bypass gástrico), la edad promedio fue de 40.6 años (33 a 44 años); el IMC promedio inicial fue de $33.14 \mathrm{~kg} / \mathrm{m}^{2}$ (30.6 a 34.5), con una pérdida media de $22 \mathrm{~kg}$ (18 a $31 \mathrm{~kg}$ ), alcanzando un IMC de $26.06 \mathrm{~kg} / \mathrm{m}^{2}$ (23.7 a 27.9). Doce pacientes presentaron diabetes mellitus, controlada en su totalidad tras el bypass gástrico (Tabla II).

En el grupo C (pacientes con obesidad GII previa a la pérdida ponderal por bypass), la edad promedio fue de 41 años (35 a 53 años); el IMC inicial fue de $37.99 \mathrm{~kg} / \mathrm{m}^{2}$ (36.1 a 39.6), con una pérdida media de $49.6 \mathrm{~kg}(36 \mathrm{a} 62 \mathrm{~kg})$, llegando a un IMC promedio de $26.54 \mathrm{~kg} / \mathrm{m}^{2}(23.8$ a 27.5). Ocho pacientes presentaron diabetes mellitus, controlada en su totalidad tras el bypass gástrico (Tabla II).

Finalmente, el grupo D (pacientes con obesidad GIII previa a la pérdida ponderal por bypass), con una edad promedio de 37.2 años (33 a 58 años); el IMC inicial fue de $50.71 \mathrm{~kg} / \mathrm{m}^{2}$ (42.3 a 70.1), con pérdida promedio de $61 \mathrm{~kg}$ (48 a $81 \mathrm{~kg}$ ) e IMC posterior de $27.67 \mathrm{~kg} / \mathrm{m}^{2}$ (26.2 a 29.9). Seis pacientes de este grupo presentaron hipertensión arterial sistémica y 8 diabetes mellitus, con- troladas en su totalidad tras el bypass gástrico (Tabla II).

Datos histológicos. (Fig. 1) Los hallazgos histológicos reflejaron que en el Grupo A no hubo ningún cambio significativo más que en 1 paciente que presentó cambios moderados en la densidad de las fibras elásticas, ninguna alteración en las fibras de colágeno y aumento moderado en el grosor del estrato córneo como indicador epidérmico.

En el Grupo B identificamos cambios moderados en la morfología de las fibras elásticas y de colágeno en la mitad de los pacientes; ningún cambio severo y el resto dentro de normalidad. El grosor epidérmico estaba aumentado en 5 pacientes y atrófico en otro.

En las biopsias del Grupo C encontramos cambios moderados (disminución de la distribución y adelgazamiento de los haces de colágeno y fibras elásticas en la dermis papilar) en 4 pacientes y cambios severos (disminución de la distribución y adelgazamiento de los haces de colágeno y fibras elásticas en la dermis reticular) en 14. Solamente 2 muestras presentaron normalidad histológica. El grosor del estrato córneo estaba significativamente engrosado en todos los pacientes, con una media de 33 líneas celulares, siendo lo normal de 20 a 30 capas celulares.

Finalmente, en el Grupo D todas las muestras presentaron cambios severos en la morfología y densidad de las fibras elásticas y de colágeno y todos presentaron engrosamiento de la epidermis, con una media de 37 líneas celulares.

Datos clínicos del sitio quirúrgico. Del total de pacientes del Grupo A, únicamente 2 presentaron conjuntamente equimosis y epidermólisis, de los cuales 1 presentó dehiscencia de $2 \mathrm{~cm}$ (Tabla III).

En el Grupo B se presentó 1 caso de equimosis aislada y 5 casos de equimosis conjunta con epidermólisis, de los cuales 3 evolucionaron a necrosis de bordes quirúrgicos y finalmente dehiscencia localizada de la herida menor de $5 \mathrm{~cm}$. En esos casos de dehiscencia pudimos

Tabla II. Media de datos demográficos de los $\mathbf{4}$ grupos de estudio

\begin{tabular}{|c|c|c|c|c|}
\hline & GRUPO A & GRUPO B & GRUPO C & GRUPO D \\
\hline $\begin{array}{l}\text { EDAD PROMEDIO } \\
\text { (años) }\end{array}$ & $39.2(29-48)$ & $40.6(33-44)$ & $41(35-53)$ & $37.2(33-58)$ \\
\hline COMORBILIDADES & $1 \mathrm{HAS}$ & $12 \mathrm{DM}$ & $8 \mathrm{DM}$ & $\begin{array}{l}6 \mathrm{HAS} \\
8 \mathrm{DM}\end{array}$ \\
\hline $\begin{array}{c}\text { IMC INICIAL } \\
\left(\mathrm{Kg} / \mathrm{m}^{2}\right)\end{array}$ & 29.23 & 33.14 & 37.99 & 50.71 \\
\hline Kg PERDIDOS & 6 & 22 & 49.6 & 61 \\
\hline $\begin{array}{l}\text { IMC POSTERIOR } \\
\left(\mathrm{Kg} / \mathrm{m}^{2}\right)\end{array}$ & 26.89 & 26.06 & 26.54 & 27.67 \\
\hline
\end{tabular}


Tabla III. Datos clínicos del sitio quirúrgico.

\begin{tabular}{|c|c|c|c|c|}
\hline & $\begin{array}{c}\text { GRUPO A } \\
\text { (Sobrepeso) }\end{array}$ & $\begin{array}{c}\text { GRUPO B } \\
\text { (Obesidad GI) }\end{array}$ & $\begin{array}{c}\text { GRUPO C } \\
\text { (Obesidad GII) }\end{array}$ & $\begin{array}{c}\text { GRUPO D } \\
\text { (Obesidad GIII) }\end{array}$ \\
\hline EQUIMOSIS ASLADA & 0 & 1 & 0 & 1 \\
\hline $\begin{array}{c}\text { EPIDERMÓLISIS } \\
\text { AISLADA }\end{array}$ & 0 & 0 & 0 & 0 \\
\hline $\begin{array}{c}\text { EQUIMOSIS } \\
\text { CONJUNTA CON } \\
\text { EPIDERMÓLISIS }\end{array}$ & 2 & 5 & 6 & 9 \\
\hline $\begin{array}{l}\text { NECROSIS } \\
\text { SECUNDARIA }\end{array}$ & 0 & $\begin{array}{c}3 \\
\text { secundaria a la equimosis } \\
+ \text { epidermólisis } \\
\text { inicial }\end{array}$ & $\begin{array}{c}4 \\
\text { secundaria a la equimosis } \\
+ \text { epidermólisis } \\
\text { inicial } \\
\end{array}$ & $\begin{array}{c}9 \\
\text { secundaria a la equimosis } \\
+ \text { epidermólisis } \\
\text { inicial }\end{array}$ \\
\hline NECROSIS AISLADA & 0 & 0 & 4 & 5 \\
\hline \multirow{3}{*}{$\begin{array}{l}\text { DEHISCENCIA } \\
\text { SECUNDARIA }\end{array}$} & \multirow{3}{*}{$\begin{array}{l}\text { secundaria a la equimosis } \\
+ \text { epidermólisis inicial }\end{array}$} & \multirow{3}{*}{$\begin{array}{l}\text { todas secundarias a la } \\
\text { equimosis }+ \text { epidermólisis } \\
\text { inicial) }\end{array}$} & $8(<5 \mathrm{~cm})$ & 14 \\
\hline & & & \multirow{2}{*}{$\begin{array}{c}4 \text { secundarias a la } \\
\text { equimosis }+ \\
\text { epidermólisis inicial } \\
4 \text { secundarias a la } \\
\text { necrosis aislada }\end{array}$} & $\begin{array}{c}10(>3 \mathrm{y}<5 \mathrm{~cm}) \\
5 \text { secundarias a la } \\
\text { equimosis }+ \text { epidermólisis } \\
\text { inicial } \\
5 \text { secundarias a la } \\
\text { necrosis aislada }\end{array}$ \\
\hline & & & & $\begin{array}{c}4(>5 \mathrm{y}<10 \mathrm{~cm}) \\
\text { todas secundarias a la } \\
\text { equimosis }+ \text { epidermólisis } \\
\text { inicial }\end{array}$ \\
\hline INFECCIÓN & 0 & $\begin{array}{c}1 \\
(E . \text { Coli }) \\
\text { equimosis + epidermólisis } \\
\text { inicial }\end{array}$ & $\begin{array}{c}2 \\
\text { (2: E.Coli) } \\
\text { equimosis + epidermólisis } \\
\text { inicial) }\end{array}$ & $\begin{array}{c}4 \\
\text { 2: E. Coli } \\
\text { 1: S. Aureus } \\
\text { 1: P. Aeuroginosa } \\
\\
\text { todas de la equimosis con } \\
\text { epidermólisis inicial }>5 \mathrm{y} \\
<10 \mathrm{~cm}\end{array}$ \\
\hline
\end{tabular}

corroborar solo 1 paciente con infección demostrada por cultivo (E. Coli) (Tabla III).

En el Grupo C se presentaron 6 casos de equimosis conjunta con epidermólisis, de los que 4 evolucionaron a necrosis del borde quirúrgico y finalmente a dehiscencia. Otros 4 casos presentaron necrosis aislada que también concluyó en dehiscencia. Estas 8 dehiscencias fueron menores de $5 \mathrm{~cm}$ y en 2 casos se identificó infección por E. Coli corroborada por cultivo. Ambos casos fueron en pacientes que presentaron inicialmente equimosis conjunta con epidermólisis. (Tabla III).

En el Grupo D documentamos 1 caso de equimosis aislada y 9 casos de equimosis conjunta con epidermólisis; esos 9 casos evolucionaron a necrosis de bordes quirúrgicos y finalmente dehiscencia localizada de la herida ( 5 casos de entre 3 y $5 \mathrm{~cm}$ y 4 casos de entre 5 y $10 \mathrm{~cm}$ ). Otros 5 pacientes presentaron dehiscencia de herida de entre 3 y $5 \mathrm{~cm}$ sin presentar otra evidencia clínica previa más que la necrosis aislada. Del total de dehiscencias pudimos corroborar 4 infecciones por cultivo (2 para
E. Coli, 1 para $S$. Aureus y 1 para P. Aeroginosa) en los casos donde las dehiscencias fueron de 5 a $10 \mathrm{~cm}$, todas de los casos con equimosis y epidermólisis inicial (Tabla III).

\section{Estadística inferencial}

Análisis estadístico. Realizamos el análisis mediante la prueba chi cuadrado y análisis de correspondencia simple, ya que las variables son cualitativas ordinales

Comparamos los 4 grupos: sobrepeso, obesidad GI, obesidad GII y obesidad GIII, de acuerdo a la densidad de fibras elásticas, morfología de las fibras elásticas, densidad de colágeno, morfología de colágeno, grosor de piel, presencia de fibroblastos, presencia de equimosis, epidermólisis, necrosis, dehiscencia de herida y presencia de infección.

La comparación entre los 4 grupos respecto a la densidad de fibras elásticas indicó una diferencia significativa, con valor $\mathrm{p}<0.0001$, entre los 4 grupos, principalmente entre sobrepeso y obesidad GIII, lo cual indica que los 
cambios en la densidad de las fibras elásticas del grupo con obesidad GIII son severos.

Al comparar la morfología de las fibras elásticas también encontramos una diferencia significativa, con valor $p<0.0001$, entre los 4 grupos, principalmente entre el grupo de obesidad GIII y sobrepeso, lo cual indica que la morfología de las fibras elásticas en el grupo de obesidad GIII está severamente alterada.

Con respecto a la densidad del colágeno también encontramos diferencias significativas entre los 4 grupos, valor $\mathrm{p}<0.0001$, principalmente entre el grupo de obesidad GIII y sobrepeso, lo que indica una densidad de colágeno severamente alterada en el grupo de obesidad GIII. En cuanto a la morfología del colágeno encontramos diferencia significativa entre los 4 grupos con valor $\mathrm{p}<0.0001$, principalmente entre el grupo de obesidad GIII frente al grupo de sobrepeso, resultado que indica daño severo en la morfología del colágeno en pacientes con algún grado de obesidad.

En el grosor de la piel registramos diferencias significativas entre los grupos con un valor $p<0.0001$. Las alteraciones en el grosor se presentan a partir de la obesidad GII y GIII. La actividad de los fibroblastos es significativamente diferente entre los pacientes con sobrepeso y los pacientes con cualquier grado de obesidad, con valor $\mathrm{p}<0.0001$.

Con respecto a las complicaciones, encontramos diferencia estadísticamente significativa en los casos de presencia de equimosis, epidermólisis, necrosis y dehiscencia, con valor de $p<0.0001$. Estas diferencias se presentan a partir de la obesidad GII y III. Dados los resultados de chi cuadrado, calculamos el riesgo relativo (RR) para cada una de estas complicaciones quirúrgicas, obteniendo que la obesidad GII aumenta 8 veces la probabilidad de presentar dehiscencia $(\mathrm{RR}=8)$, mientras que la obesidad GIII aumenta 5 veces la probabilidad de presentar equimosis y epidermólisis (en ambos casos el RR es igual a 5), aumenta 9 veces la probabilidad de presentar necrosis $(\mathrm{RR}=9)$ y 14 veces la probabilidad de presentar dehiscencia $(\mathrm{RR}=14)$.

No obtuvimos diferencia estadísticamente significativa para la presentación de infecciones entre los 4 grupos de pacientes.

\section{Discusión}

El tratamiento quirúrgico de la obesidad mediante bypass gástrico está indicado a partir de un IMC mayor de $40 \mathrm{~kg} / \mathrm{m}^{2}$ (obesidad GIII / obesidad mórbida) o en los grados menores de obesidad (GI y II / IMC mayor de 30 y $35 \mathrm{~kg} / \mathrm{m}^{2}$ respectivamente) que presentan comorbilidades asociadas de difícil control, como diabetes me- llitus, sin respuesta al tratamiento higiénico-dietético. ${ }^{(13)}$ En dichos pacientes, tras realizar un bypass gástrico se logra el control, la mejoría o la remisión completa de la enfermedad, pero al mismo tiempo se presentan grandes secuelas cutáneas, motivo por el cual son enviados a nuestro Servicio para realizar reconstrucción por segmentos corporales.

Para iniciar dicho proceso, todos los pacientes tratados en nuestro Servicio deben alcanzar un IMC menor de $28 \mathrm{~kg} / \mathrm{m}^{2}$ cuando presentaron obesidad GI y II, y menor de $31 \mathrm{~kg} / \mathrm{m}^{2}$ los de GIII / obesidad mórbida, así como mantener dicha estabilidad por un periodo de al menos 3 meses. ${ }^{(14)}$ Además, deben continuar desde el postoperatorio inmediato el régimen nutricional implementado previamente por el Servicio de Nutrición Clínica, basado en mantener en niveles óptimos los nutrientes esenciales mediante multivitamínicos más minerales diarios que contengan hierro, ácido fólico y tiamina; 1.200 a $1.500 \mathrm{mg}$ de calcio elemental en la dieta y como citrato de calcio en suplementos; al menos 3.000 unidades internacionales de vitamina $D$; vitamina $B 12$ necesaria para mantener niveles en rango normal; hierro mediante multivitamínicos y suplemento adicional que aporte de 40 a $60 \mathrm{mg}$ por día. (15,16) Por otro lado, siguiendo el protocolo de la evaluación preoperatoria, deben aprobar también la evaluación del Servicio de Psicología que corrobore un adecuado apoyo familiar y un firme compromiso del paciente para llevar a cabo las funciones correspondientes en su cuidado postoperatorio. ${ }^{(17)}$

Sin embargo, a pesar de seguir todo el protocolo preoperatorio descrito, en un paciente postpérdida masiva de peso aún identificamos un alto porcentaje de complicaciones tanto locales como sistémicas en nuestra población, ${ }^{(14)}$ y en otras series internacionales publicadas, ${ }^{(18)}$ lo cual no ocurre en los pacientes promedio convencionales que presentan únicamente sobrepeso, como en el grupo control de este estudio, que se sometieron a una pérdida ponderal previa a la abdominoplastia únicamente a base de dieta y ejercicio. ${ }^{(10)}$

Así pues, se estima que en un paciente con sobrepeso que se someterá a una abdominoplastia, se planificará una pérdida ponderal de aproximadamente un $5 \%$ de su peso total para llegar a su peso ideal, lo que representa un promedio de entre 4 y $9 \mathrm{~kg}$ del exceso de peso. Ahora bien, si se compara con los pacientes que ya se encuentran en un grado de obesidad, estos perderán en promedio un 15,20 y $30 \%$ de su peso total antes de llegar a un peso estable que los haga candidatos a una reconstrucción abdominal. Esto significa una pérdida de entre 15 y hasta $60 \mathrm{~kg} \cdot{ }^{(10-12)}$

Los cambios histológicos dérmicos que se presentan tras la pérdida de peso están relacionados con una degra- 
dación de las fibras elásticas y de colágeno que habitualmente se inicia en etapa adulta tardía o en la senectud, pero que, en los pacientes con obesidad se acelera. Nos referimos entonces a que se produce un envejecimiento cutáneo prematuro. ${ }^{(19)}$

Así pues, el alto porcentaje de complicaciones que se producen en la herida quirúrgica de los pacientes que padecieron obesidad, se encuentran directamente relacionadas con el máximo grado de tensión cutánea que presentaron: a mayor obesidad, mayor deterioro. Si bien este dato está documentado de cierta forma en series internacionales en las que se habla sobre las complicaciones generales de la cirugía reconstructiva tras pérdida masiva de peso, estas únicamente mencionan que el resultado es atribuible a un mayor índice de masa corporal o al impacto de la deficiencia nutricional, pero no especifican que dichas complicaciones del sitio quirúrgico están relacionadas también con los cambios histológicos. ${ }^{(18,20)}$

La importancia del presente estudio radica en que pudimos obtener, en grupos homogéneos, el RR de presentar una complicación del sitio quirúrgico de acuerdo al IMC previo a la pérdida ponderal, bien haya sido por medidas higiénico-dietéticas en el grupo de sobrepeso o por bypass gástrico en los grupos de obesidad GI, II y III. Como pudimos evidenciar, el RR de las complicaciones locales, principalmente de la dehiscencia de la herida, se eleva 8 veces en la obesidad GII y hasta 14 veces en la obesidad GIII, haciendo mención especial a que en nuestra serie no hubo significancia estadística en relación a la infección del sitio quirúrgico.

No existen estudios que evidencien cuál es el RR de un paciente operado de bypass gástrico en comparación con un paciente convencional que perdió pocos kilos antes de ser sometido a una abdominoplastía; únicamente se han descrito, en las series más grandes, cuáles son las principales complicaciones presentadas pero no se emite una recomendación en relación al por qué de la diferencia de morbilidad tan alta. ${ }^{(18,20)}$

Es por ello que, basándonos en los resultados de nuestro estudio, resaltamos la importancia de que a pesar de realizar un adecuado protocolo perioperatorio, con una correcta selección de pacientes, meta de IMC alcanzada de acuerdo al grado de obesidad previa, correcta estabilización de las deficiencias nutricionales, estado psicológico estable y técnica quirúrgica estandarizada tanto en el grupo de pacientes con sobrepeso (abdominoplastia convencional), como en los grupos de obesidad (despegamiento limitado a las incisiones de la flor de lis, libres de tensión en el cierre), las dehiscencias se van a presentar en un mayor porcentaje debido a los cambios histológicos cutáneos que ocurren tras un bypass gástrico. ${ }^{(10-11-18-20)}$
Cuando nos enfrentamos a un paciente postbariátrico debemos extremar las medidas antes, durante y después del procedimiento quirúrgico, para de esta forma evitar un incremento del riesgo. Al tener estandarizados todos los pacientes incluidos en el estudio, las limitaciones para la obtención de resultados están relacionadas con el aspecto del cuidado personal de los pacientes una vez que son egresados de la unidad hospitalaria: cuidados de las heridas en casa, actividades realizadas, alimentación adecuada, factores que también interfieren directamente en los hallazgos identificados en las consultas posteriores a la cirugía.

Por ello, a partir de este trabajo, podemos partir hacia nuevas líneas de investigación en relación a incrementar la severidad de la evaluación psicológica previa a la cirugía, así como realizar escalas de función postoperatoria donde identifiquemos las actividades que el paciente realizó y que pudieron influir en su recuperación general y sobre todo, en la evolución del sitio quirúrgico.

\section{Conclusiones}

Los cambios histológicos y la degeneración dérmica se presentan en todos los pacientes que presentaron algún grado de obesidad y que se sometieron a un bypass gástrico, en contra de lo que ocurre con los pacientes que únicamente presentan sobrepeso, pues tras una pérdida ponderal con dieta y ejercicio, no presentan dichas alteraciones en la piel. Es por ello que en el momento de someterse a un procedimiento quirúrgico, como una abdominoplastia en el caso del presente estudio, los pacientes postbariátricos que tuvieron obesidad Grado II presentaron un riesgo relativo 8 veces mayor de tener una dehiscencia del sitio quirúrgico y 14 veces mayor en el Grado III.

Así pues, teniendo conocimiento de este riesgo relativo, todo paciente postbariátrico debe ser estrictamente protocolizado durante todo el periodo perioperatorio por un equipo multidisciplinario, y por nuestra parte, poner un mayor énfasis en el grado de obesidad previa presentado, pues este tendrá un impacto directamente proporcional en la presencia de complicaciones durante la cirugía de reconstrucción.

\section{Dirección del autor}

Dr. José Martín Morales Olivera

Hospital General Tiáhuac

Avda. La Turba 655

Col. Villa Centroamericana CP 13250

Del. Tiáhuac, Ciudad de México, México

Correo electrónico: martinmo10@hotmail.com 


\section{Bibliografía}

1.- Gupta N, Goel K, Shah P et. Al. Childhood Obesity in Developing Countries: Epidemiology, Determinants, and Prevention. Endocr Rev. 2012; 33(1):48-70.

2.- Gilbert EW, Wolfe BM. Bariatric Surgery for the Management of Obesity: State of the field. Plast Reconstr Surg. 2012; 130(4): 948-954.

3.- Greco JA, Castaldo ET, Nanney LB et. al. The effect of weight loss surgery and body mass index on wound complications after abdominal contouring operations. Ann Plast Surg. 2008; 61(3): 235-242.

4.- Sami K, Elshahat A, Moussa M et.al. Image analyzer study of the skin in patients with morbid obesity and massive weight loss. Op Acc Journal of Plastic Surg. 2015; 15: 17-28.

5.- Halawi A, Abiad F, Abbas O. Bariatric surgery and its effects on the skin and skin diseases. Obes Surg. 2013; 23: 408-413.

6.- Orpheu SC, Coltro PS, Scopel GP, et al. Collagen and elastic content of abdominal skin after surgical weight loss. Obes Surg. 2010;20:480-486.

7.- Erdmann D. Letter to editor: Dermal Histomorphology in postbariatric patients. Ann Plastic Surg. 2013; 71(4): 441-442.

8.- Kumar N, Kumar P, Praaad K et.al. A histological study on the distribution of dermal collagen and elastic fibres in different regions of the body. Int J of Med Med Sci. 2012; 4(8):171-176.

9.- Fearmonti R, Blanton $\mathbf{M}$, Bond $\mathbf{J}$ et.al. Changes in dermal histomorphology following surgical weight loss versus diet induced weight loss in the morbidly obese patient. Ann Plast Surg. 2012; 68(5): 507-512.

10.- RichterD, StoffA.Abdominoplasty procedures. NeliganP,Plastic Surgery. Philadelphia, United States of America: Elsevier, 2013.
11.- Saldanha OR, Salles AG, Ferreira M et.al. Aesthetic evaluation of lipoabdominoplasty in overweight patients. Plast Reconstr Surg. 2013; 132 (5): 1103-1112.

12.- Nahabedian MY. Discussion: Aesthetic evaluation of lipoabdominoplasty in overweight patients. Plast Reconstr Surg. 2013; 132: 1113-1114.

13.- Espinosa O, Pineda O, Maydón HG, et al. Type 2 diabetes mellitus outcomes after laparoscopic gastric bypass in patients with BMI $<35 \mathrm{~kg} / \mathrm{m}^{2}$ using strict remission criteria: early outcomes of a prospective study among Mexicans. Surg Endosc. 2018; 32(3):1353-1359.

14.- Portes A; Morales-Olivera JM, Corona $\mathrm{R}$ et.al. Interdisciplinary clinical guidelines for massive weight loss patients during plastic reconstructive surgery; Cir plást iberolatinoam 2016; 42(1): 78-91.

15.- Naghshineh N, Rubin JP. Preoperative evaluation of the body contouring patient: the cornerstone of patient safety. Plastic Surg. 2014 (41) 637-643.

16.- Bossert RP, Rubin JP. Evaluation of the weight loss patient presenting for plastic surgery consultation. Plast Reconstr Surg. 2012; 130 (6): 1363-1365.

17.- Pappas, T.N. Physiological satiety implications of gastrointestinal antiobesity surgery. American J Clin Nutrition 1992; 55: 571-572.

18.- Coon D, Gusenoff J, Kanaan $\mathbf{N}$ et.al. Body mass and surgical complications in the postbariatric reconstructive patient: analysis of 511 cases. Ann Surg. 2009; 249(3): 397-401.

19.- Lindsted E, Sandblom P. Wound Healing in man; Tensile Strength of Healing Wounds in Some Patient Groups. Ann Surg. 1975; 181(6):842-847.

20.- Agha-Mohammadi S. Hurwitz DJ. Potential impacts of nutritional deficiency of postbariatric patients on body contouring patients. Aesth Plast Surg. 2010 (34):617-625.

\title{
Eomentario al artículo “Cambios histológicos dérmicos tras pérdida masiva de peso y su repercusión en la herida quirúrgica en abdominoplastia"
}

\author{
Linda L. RINCÓN RUBIO \\ Cirujano Plástico, Centro Diagnóstico Docente las Mercedes, Directora de la Unidad de Cirugía Plástica y Postbariatrica Ca- \\ racas (Plásticabariátrica), Profesora Universitaria Hospital Carlos J. Bello Cruz Roja Venezolana, Caracas, Venezuela. \\ Directora del Capítulo de Cirugía Postbariátrica de la FILACP 2018-2020
}

Queridos colegas, ante todo felicitarles por su labor de investigación en la población de pacientes postbariátricos que cada día aumentan en número y que definitivamente ofrecen retos diferentes a los de la Cirugía Plástica convencional. Cuando tratamos la estructura cutánea modificada por obesidad previa en estos grupos de pacientes y evidenciamos clínicamente mayor número de complicaciones, definitivamente tiene que haber características especiales y específicas que nos expliquen las razones y nos ayuden a entender mejor cómo solventar dichas complicaciones.

Su trabajo estudia las variables histológicas en cuanto a cambios de la densidad de fibras elásticas, cambios de morfología de las fibras elásticas, cambios de la densidad del colágeno, cambios de morfología del colágeno, grosor de epidermis y signos clínicos en la piel como equimosis, epidermólisis, necrosis cutánea, dehiscencia de herida e infección. Comparan 4 grupos diferentes de pacientes y correlacionaron los hallazgos histológicos con los signos clínicos. Logran así determinar que existe evidente relación de mayor daño tisular cuanto mayor es el índice de obesidad, lo cual se traduce más a un efecto "mecánico" del estrato dermograso sometido a grandes estiramientos y a engrosamiento de sus estructuras.

Sin embargo, más allá de los procesos "mecánicos" que suceden en el estrato dermograso ${ }^{(1)}$ de los pacientes con cualquier grado de obesidad, que claramente repercuten en la calidad de los tejidos y tienen sus consecuencias en las complicaciones quirúrgicas, ${ }^{(2)}$ existen evidencias de cambios estructurales generados por la propia pérdida masiva de peso que nosotros pudimos comprobar en nuestra investigación al respecto publicada en esta misma revista, ${ }^{(3)}$ como son la presencia de inflamación crónica perianexial de carácter crónico, fibrosis tipo esclerosis subepitelial y necrosis grasa, que nos pueden explicar el estado de stress sostenido de los tejidos en 
este grupo de pacientes y que conllevan la aparición de hipoxia, neovascularización reparativa y daño tisular preexistente..$^{(3)}$ Es decir, no solo interviene el efecto mecánico que ustedes bien describen, sino que existen cambios adaptativos celulares en respuesta a la gran pérdida ponderal (mayor de $40 \mathrm{~kg}$ ) que, sumado a lo que ustedes magistralmente han observado, nos lleva a concluir que en definitiva esta población de pacientes postbariátricos son biomecánicamente diferentes, histológicamente diferentes y quizás hasta inmunológicamente diferentes, dando así pie a próximos estudios de investigación que nos ayuden a comprender mejor sus debilidades y a prepararnos todos como cirujanos postbariatricos, cada día mejor, para vencer todas estas dificultades.

Felicitaciones.

\section{Bibliografía}

1.- Fearmonti R, Blanton $\mathbf{M}$, Bond $\mathbf{J}$ et.al. Changes in dermal histomorphology following surgical weight loss versus diet induced weight loss in the morbidly obese patient. Ann Plast Surg. 2012; 68(5): 507-512.

2.- Greco JA, Castaldo ET, Nanney LB et. al. The efect of weight loss surgery and body mass index on wound complications after abdominal contouring operations. Ann Plast Surg. 2008; 61(3): 235-242.

3.- Rincón Rubio L, Cemboraín Valarino M, et al. Evaluación de cambios histopatológicos secundarios a cirugía bariátrica. Cir. plást.iberolatinoam. 2018, 44 (4): 379-387.

\section{Respuesta al comentario de la Dra. Rincón Rubio}

\section{José Martín MORALES-OLIVERA}

Querida colega, agradecemos enormemente los comentarios y las atinadas observaciones que le has dedicado a nuestro trabajo.

Como bien lo mencionaste, tras el aumento de la cirugía bariátrica como tratamiento quirúrgico de la obesidad, la población de pacientes con secuelas de pérdida masiva de peso es cada día mayor en todo el mundo y por ello requiere un mayor estudio.

Las recomendaciones generales de estos pacientes ya se han establecido, y consisten en completar estrictamente el protocolo perioperatorio del equipo multidisciplinario; no escatimar en medidas de seguridad, y realizar un abordaje quirúrgico más conservador ${ }^{(1)}$.

Sin embargo, existe poca literatura que mencione las causas (histológicas, mecánicas y/ó inmunológicas), del porque el incrementado número de complicaciones en esta población en particular.

Muchos de los estudios existentes comparan y reportan las diferencias generales entre grupos de pérdida masiva de peso mediante un procedimiento bariátrico, frente a grupos de pérdida con dieta y ejercicio, atribuyendo dichos resultados a las deficiencias nutricionales, pero sin mencionar causas histológicas o un riesgo relativo de acuerdo con su grado inicial de obesidad. ${ }^{(2)}$
Es por ello que, al revisar también tu publicación, podemos afirmar que el conjunto de ambas investigaciones logra un gran ensamble que nos lleva a una conclusión clínica - histológica más firme y que refuerza la premisa de que: "un paciente postbariátrico nunca será igual a un paciente estético convencional".

Con lo anterior reiteramos también que estamos frente a una rama de la Cirugía Plástica que merece mención aparte y en la cual aún falta mucho por aprender, mucho por descubrir, y mucho por describir, pero con la seguridad de que la tenacidad de todos los que nos dedicamos a tratar estos pacientes cotidianamente, seguiremos las líneas de investigación faltantes con las que lograremos establecer protocolos universales.

Nuevamente colega, infinitas gracias.

\section{Bibliografía}

1.- Shermak M. Pearls and perils of caring for the postbariatric body contouring patient. Plast Reconstr Surg. 2012: 585e-596e.

2.- Gusenoff JA, Coon D, Rubin JP. Implications of weight loss method in body contouring outcomes. Plast Reconstr Surg. 2009: 373-376. 
